\title{
Ammonia decomposition over cobalt/carbon catalysts - effect of carbon support and electron donating promoter on activity
}

\author{
Laura Torrente-Murciano ${ }^{*}, \mathbf{a}$, Alf K. Hill ${ }^{\mathrm{b}}$ and Tamsin E. Bell ${ }^{\text {a }}$ \\ ${ }^{a}$ Department of Chemical Engineering and Biotechnology, University of Cambridge, \\ Cambridge, CB2 3RA, UK \\ ${ }^{b}$ Department of Chemical Engineering, University of Bath, Bath, BA2 7AY, UK
}

\begin{abstract}
This paper sets the new design parameters for the development of low temperature ammonia decomposition catalysts based on readily available cobalt as an alternative to scarce but highly active ruthenium-based catalysts. By using a variety of carbon materials as catalytic supports, we systematically demonstrate that microporous supports capable of stabilising small cobalt crystallites $(\sim 2 \mathrm{~nm}$ ) lead to high catalytic activities compared to bigger nanoparticles. Additionally, the degree of graphitisation of the carbon support has a detrimental effect on the activity of the cobalt (0) active sites, likely due to their potential as electron donator. Consequently, the addition of electron donating promoters such as cesium substantially decreases the activity of the cobalt catalysts. This relationship deviates from the trends observed for ruthenium-based catalysts with an optimum 3-5 nm size where an increase of the graphitisation degree of the support and the addition of electron donating promoters increases the ammonia decomposition activity.
\end{abstract}

Keywords: ammonia decomposition; $\mathrm{H}_{2}$ generation, cobalt, ruthenium, carbon

\footnotetext{
* Corresponding author: Laura Torrente-Murciano, Phone: +44 122376 8664, Email: 1t416@cam.ac.uk
} 


\section{Introduction}

Hydrogen is commonly presented as a promising alternative to fossil fuels in what is called the hydrogen economy. At the moment, there are few prototypes in the market for the use of hydrogen as road transportation fuel for proton exchange membrane fuel cells (PEM-FC) [1, 2]. Although its widespread implementation would facilitate the reduction of emissions at the point of use, with associated beneficial environmental impacts, there are current concerns about the safe transportation and storage of hydrogen $[3,4]$ due to its high flammability. This has triggered the scientific community to develop novel hydrogen storage technologies, mainly based on porous materials $[3,5]$. However, none of them currently fulfil the 9 wt. $\%$ gravimetric hydrogen storage target established by the US Department of Energy in 2015 [6]. Within this context, ammonia is presented as an attractive carbon-free energy vector molecule with a hydrogen content of $17.3 \mathrm{wt} . \%$, higher than liquid hydrogen on both a volumetric and gravimetric basis [7]. Additionally, ammonia has a relatively narrow combustion range of 16$25 \%$ in air, compared with $4-75 \%$ for $\mathrm{H}_{2}$ [8]. However, the feasibility of ammonia as an energy vector relies on the ability to decompose it to release hydrogen (Reaction 1) at a temperature similar to the operation of the PEM fuel cell (below 150-180 ${ }^{\circ} \mathrm{C}$ ).

$$
2 \mathrm{NH}_{3}(\mathrm{~g}) \leftrightarrows 3 \mathrm{H}_{2}(\mathrm{~g})+\mathrm{N}_{2}(\mathrm{~g})
$$

Under these relatively low temperature conditions, the decomposition of ammonia reaction is kinetically limited by the recombinative desorption of nitrogen adatoms from the metal sites [9]. Previous studies have demonstrated a volcano-type relationship between the ammonia decomposition rate of different metals as a function of their nitrogen binding energy, where the optimum nitrogen binding energy falls in the range of $544-586 \mathrm{~kJ} \mathrm{~mol}^{-1}$, which corresponds to ruthenium [9]. Indeed, the most active and extensively studied catalysts reported in the literature for the decomposition of ammonia are based on ruthenium $[10,11]$. The activity of ruthenium-based catalysts, especially at low temperatures, can be further 
enhanced by the use of electron donating promoters such as alkali metals [12] and highly conductive supports such as graphitised carbon nanotubes [13]. Despite this, the cost and scarcity of ruthenium has, in the last few years, generated a renewed interest in the development of ammonia decomposition catalysts based on alternative, readily available nonnoble metal catalysts including iron $[14,15]$, cobalt $[11,16,17]$ and nickel $[18,19]$. Amongst these, the nitrogen biding energy of cobalt is the closest one to the optimum value range. Indeed, Co/CNT catalyst prepared by incipient wetness impregnation shows superior activity to that of Ni/CNT and Fe/CNT [20]. A recent kinetic study by Lendzion-Bielun et al. [21] showed a lower ammonia decomposition activation energy on cobalt than on iron-based catalysts. Previous studies suggest that the catalytic activity of cobalt-based catalysts can be related to $i$. the level of dispersion and crystallite size [22] and ii. the cobalt-support interaction [17]. However, a recent review of the decomposition of ammonia decomposition using non-noble metals [23] reveals the lack of systematic investigation on the development of these catalysts and the need of fundamental understanding of the effects of catalyst parameters on the resulting activity. By using a range of carbon supports with different physical and chemical properties, this paper reveals the effect of metal dispersion, metal particle size, support porosity and the electronic modification of the active sites of cobalt/carbon catalysts on the decomposition of ammonia catalytic activity, providing guidelines for future catalyst development in the field.

\section{Experimental procedure}

Catalysts were prepared by incipient wetness impregnation using $\mathrm{Co}\left(\mathrm{NO}_{3}\right)_{2} \cdot 6 \mathrm{H}_{2} \mathrm{O}$ as metal precursor on various carbon supports, including multi-walled carbon nanotubes (CNT), activated charcoal and MESO-C (from Sigma Aldrich) and Ax-21 and MSC-30 (from Kansai 
Coke and Chemicals ltd). Acetone was used as metal solvent in preference of water as the lower surface tension of acetone allows for greater pore filling of the support [24]. The different supports were dried in a vacuum oven at $80^{\circ} \mathrm{C}$ overnight prior determination of their respective wetness volume by adding acetone dropwise until wetness saturation is reached. The wetness impregnation volumes were estimated as $10.1,1.9,1.7,3.0$ and $2.8 \mathrm{~mL} \mathrm{~g}^{-1}$ for CNT, activated charcoal, MESO-C, AX-21 and MSC-30 respectively. $\mathrm{CsOH} \cdot \mathrm{xH}_{2} \mathrm{O}$ and $\mathrm{Ru}(\mathrm{NO})\left(\mathrm{NO}_{3}\right)_{3}$ were used as cesium and ruthenium precursors in the promoted and reference catalysts respectively. The required amount of metal precursor was dissolved in the respective wetness volume and the different supports were impregnated dropwise. In the case of promoted catalysts, cesium was impregnated sequentially after drying the catalysts after cobalt impregnation. After synthesis, the catalysts were dried for 3 hours at $80^{\circ} \mathrm{C}$ under vacuum to remove the solvent. Catalysts were reduced under pure hydrogen flow for three hours at $400^{\circ} \mathrm{C}$.

Nitrogen adsorption analyses were carried out at $-196^{\circ} \mathrm{C}$ using a Micromeritics ASAP 2020 instrument. The surface area was calculated using the Brunauer, Emmett and Teller (BET) method. A JEOL TEM -2100 $200 \mathrm{kV}$ ultra-high resolution transmission electron microscope was used for imaging of the samples. Samples were prepared by dispersing the samples in ethanol $\left(0.5 \mathrm{mg} \mathrm{mL}^{-1}\right)$. A drop of the dispersion was added to a Lacey carbon-coated copper mesh grid and dried under vacuum. Temperature programme reduction (TPR) experiments were carried out in a Micromeritics Autochem II instrument. The samples were degassed at $400{ }^{\circ} \mathrm{C}$ under flowing argon for 20 minutes prior to TPR analysis up to $1000{ }^{\circ} \mathrm{C}$ using a temperature ramp rate of $10{ }^{\circ} \mathrm{C} \mathrm{min}^{-1}$ under $30 \mathrm{~mL} \mathrm{~min}^{-1}$ flow of $5 \% \mathrm{H}_{2} /$ Ar. Raman analyses were carried out using a Renishaw inVia Raman microscope using a $532 \mathrm{~nm}$ green Renishaw Diode Laser. 
Ammonia decomposition reactions were carried out in a continuous packed bed flow differential reactor situated inside a Carbolite tubular oven equipped with a PID control with a gas hourly space velocity of $5,200 \mathrm{ml}_{\mathrm{NH} 3} \cdot \mathrm{g}_{\mathrm{cat}}{ }^{-1} \cdot \mathrm{h}^{-1}$ using $25 \mathrm{mg}$ of catalyst diluted in a $4 \mathrm{~cm}^{3}$ silicon carbide bed. The inlet flow of the reactor was controlled with mass flow controllers. All the pipes were heated to $60{ }^{\circ} \mathrm{C}$ to avoid any ammonia condensation and consequent corrosion. During each catalytic study, the reaction temperature was ramped from around 200 to $550{ }^{\circ} \mathrm{C}$ at $2.5{ }^{\circ} \mathrm{C} \min ^{-1}$. The reaction temperature was measured using a thermocouple situated over the catalytic bed to avoid any temperature lag. The outlet flow of the reactor at different reaction temperatures was analysed on-line using a gas chromatograph fitted with a Hayesep Q column and a thermal conductivity detector. The mass balance closure precision was within $\mathrm{a} \pm 7 \%$ error.

\section{Results and discussion}

Cobalt was supported on a range of carbon materials by the incipient wetness impregnation method and tested for the catalytic production of hydrogen via the ammonia decomposition reaction. The conversion profiles with respect to reaction temperature of the different systems are shown in Error! Reference source not found.a. The catalytic profile of ruthenium nanoparticles supported on carbon nanotubes is also shown as reference. It is important to note that all the studied catalysts were stable for up to six consecutive reactions ranging from room temperature to $500^{\circ} \mathrm{C}$. For any given reaction temperature, cobalt supported on $\mathrm{Ax}-21$ shows the highest activity. A slightly lower activity is observed with cobalt supported on MSC-30 and MESO-C catalysts, followed by CNTs. Cobalt supported on activated charcoal was found to have negligible activity within the studied temperature range. 

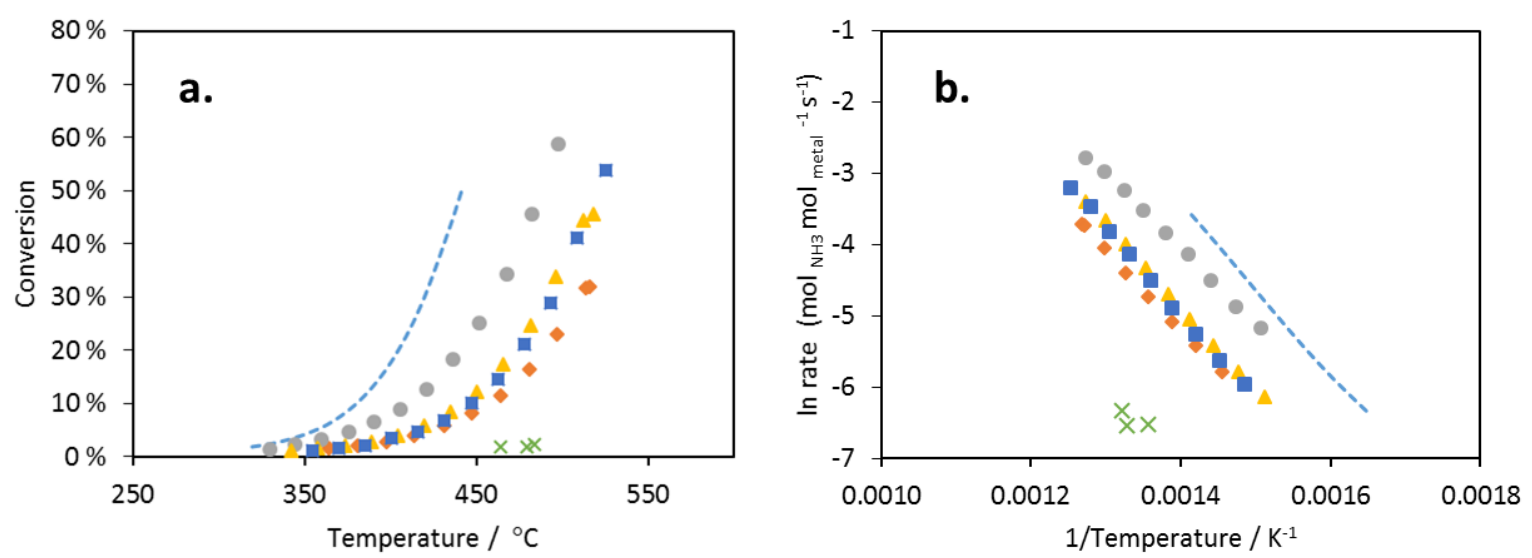

Figure 1: (a) Ammonia decomposition conversion as a function of reaction temperature of 7 wt.\%

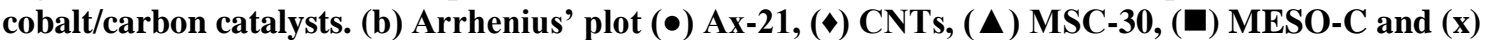
Activated charcoal. The dashed line represents the activity for $7 \mathrm{wt}$ \% $\%$ Ru/CNTs as reference catalyst. All catalysts were pre-reduced in-situ at $400^{\circ} \mathrm{C}$.

Carbon nanotubes have been shown to be the optimum carbon support for ruthenium nanoparticles due to their high conductivity which promotes the electron transfer into the ruthenium active sites [12], favouring the associative nitrogen desorption, known to be the rate-limiting step, especially at low temperatures [25]. Indeed, the catalytic activity of ruthenium substantially decreases when supported on Ax-21 (Figure 2) compared to CNTs. This observation is in agreement with a previous study where it is shown that CNTs increase the activity of ruthenium nanoparticles on the ammonia decomposition compared to other porous carbons [26]. However, the opposite is true for cobalt-based catalysts which become substantially more active when supported on Ax-21 compared to CNTs. In addition, Co/Ax21 catalyst shows a comparable activity to the Ru/Ax-21 one (Figure 2). Indeed, cobalt catalysts supported on different porous carbons exceed the activity of cobalt on CNTs although nitrogen desorption is also the rate-limiting step in these systems [11]. Further characterisation of the carbon supports and the cobalt-based catalysts demonstrates the importance of other physical and chemical carbon properties in the resulting ammonia 
decomposition catalytic activity, which do not necessarily align with the effects observed on ruthenium-based catalysts.

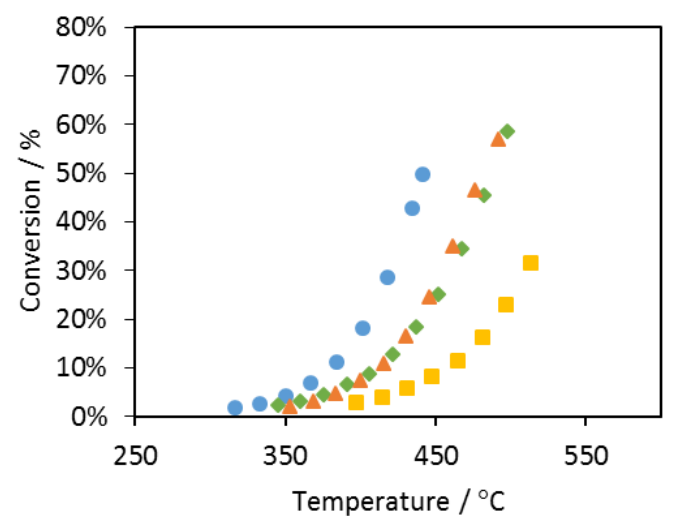

Figure 2: Ammonia decomposition conversion as a function of reaction temperature of $\bullet 7 \mathrm{wt} . \% \mathrm{Ru} / \mathrm{CNT}$, $\triangle 7$ wt.\%Ru/Ax-21, 7 wt.\% Co/Ax-21 and 7 wt.\% Co/CNT.

Raman spectroscopy was used to quantify the degree of graphitisation of the different carbon supports (Figure 3). The D band which appears at approximately $1350 \mathrm{~cm}^{-1}$ reflects the degree of disorder in the carbon structures. By contrast, the $G$ and $G$ ' bands, that occur at approximately 1600 and $2750 \mathrm{~cm}^{-1}$ respectively, reflect the graphitic ordering and alignment of the carbon structure. The degree of graphitisation of carbon materials is often described using the ratio of the intensity of $\mathrm{D}$ and $\mathrm{G}$ bands [26], which are shown in Table 1 . The $\mathrm{I}_{\mathrm{D}} / \mathrm{I}_{\mathrm{G}}$ ratio is related to the orderliness of the graphite structure and is inversely proportional to the microcrystalline planar size, which corresponds to the in-plane dimension of a single microcrystallite in graphite [26]. According to this parameter, the degree of graphitisation of the different carbon supports follows the trend of MESO-C > Activated charcoal > MSC-30 > CNTs $>$ Ax-21. 


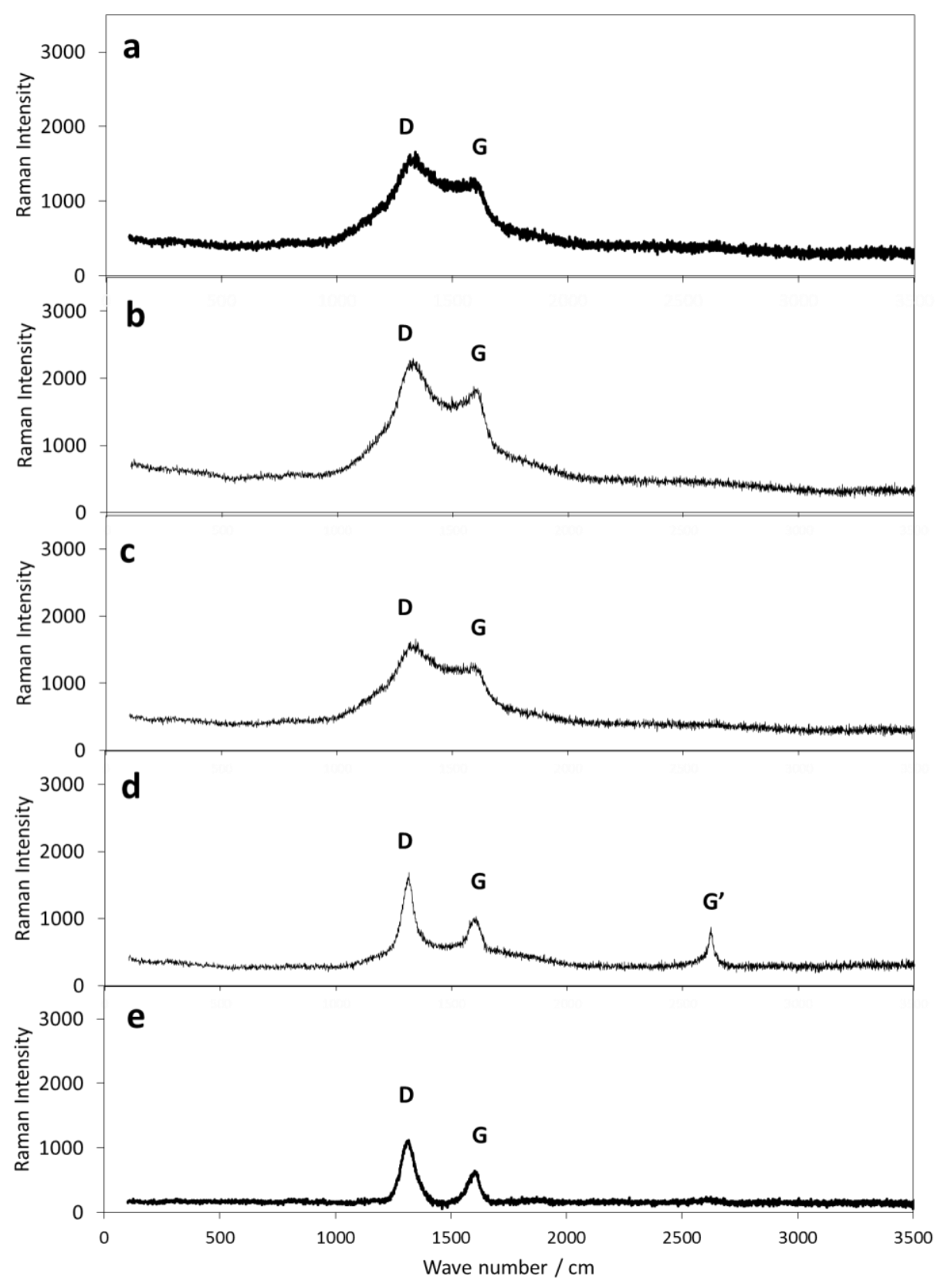

Figure 3: Raman spectra of the different carbon supports with characteristic $D, G$ and G' bands identified. a. CNTs, b. Ax-21, c. MSC-30, d. MESO-C and e. Activated charcoal.

Surprisingly, the activity for ammonia decomposition of the cobalt/carbon catalysts does not seem to correlate to the degree of graphitisation of the support (Figure 4a). Indeed, an indirect relationship between catalytic activity and $1 / \mathrm{I}_{\mathrm{D}} / \mathrm{I}_{\mathrm{G}}$ ratio is observed for all the catalysts with the exception of $\mathrm{Co} / \mathrm{MESO}-\mathrm{C}$. This is not consistent with the results observed for rutheniumbased catalysts where an increase of the graphitisation degree of the support correlates to a 
beneficial effect on their respective catalytic activity due to the facilitation of the nitrogen associative desorption step $[13,26]$. This observation is also in agreement with the work by Zhang et al. [16] who showed that cobalt supported on graphite and single-wall CNTs catalysts present low ammonia decomposition activity despite the high graphitisation structure and excellent electron transfer properties of these supports.

In addition, the activation energy of the different cobalt/carbon catalysts seems to be indirectly related to the degree of graphitisation of the support (Figure 4b), opposite to the trend previously reported for ruthenium-based catalysts [13] suggesting that other factors beyond the electronic properties of the support, greatly contribute to the low temperature activity of cobalt on the ammonia decomposition reaction.
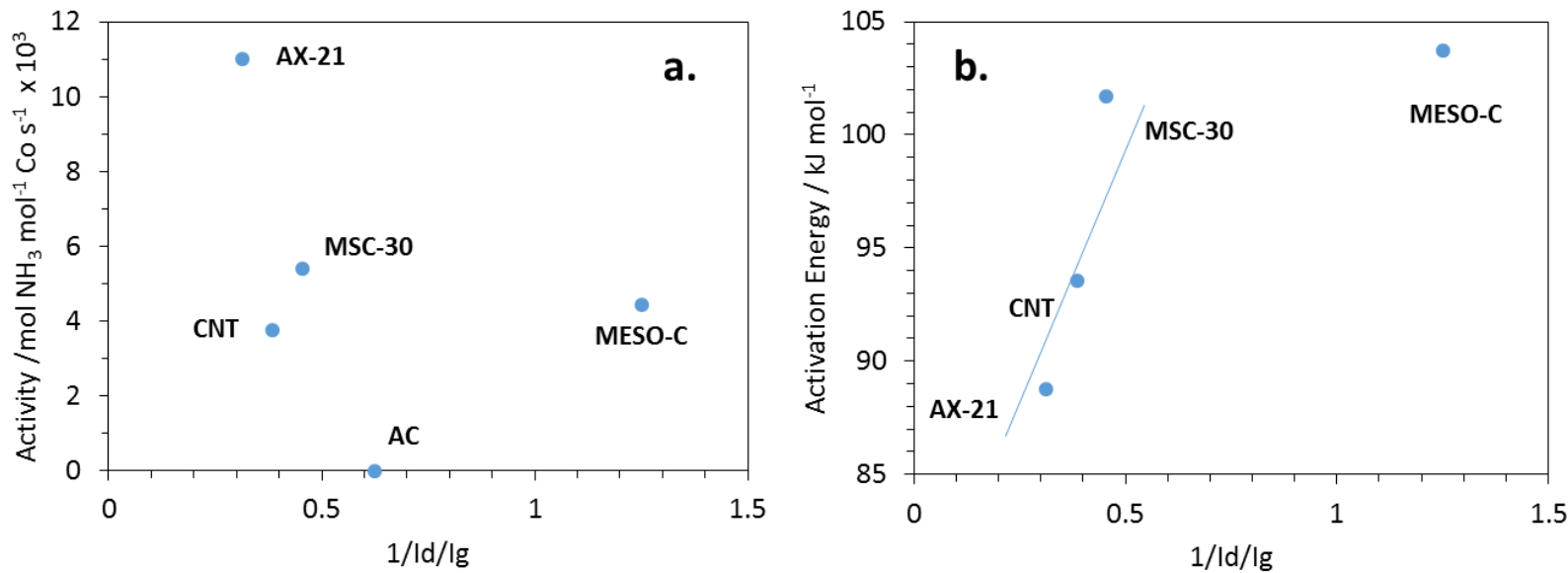

Figure 4: Relationship between the carbon support graphitic degree and a. the activity and b. activation energy of 7 wt. \% cobalt supported on carbon catalysts.

Nitrogen physisorption isotherms at $-196^{\circ} \mathrm{C}$ of the different carbon supports and their corresponding BJH dV/dlog(D) pore size distribution are shown in Figure 5. Data is presented up to a pore size of $20 \mathrm{~nm}$, with most of the distributions showing a sharp characteristic nitrogen peak at around $4 \mathrm{~nm}$ caused by the instability of the hemispherical meniscus for nitrogen desorption in pores of this specific size [27]. Ax-21 and MSC-30 carbons show 
IUPAC Type I isotherms typical of microporous material with average pore sizes of $2.3 \mathrm{~nm}$.

The $\mathrm{N}_{2}$ adsorption isotherms corresponding to the CNT and MESO-C supports show variations of the IUPAC Type IV isotherms with hysteresis loops, characteristic of mesoporous materials. CNTs show an average pore size of $27.5 \mathrm{~nm}$ representative of the tubular structure and a smaller peak at $3-4 \mathrm{~nm}$ that may be related to defects on the carbon structure. MESO-C material has an average pore size of $6.9 \mathrm{~nm}$. In addition to the mesoporosity, activated charcoal shows some significant adsorption and desorption at low relative pressure indicating a greater degree of microporosity, with an average pore size of 5.7 $\mathrm{nm}$. As expected, the surface area of the carbons is in accordance with the isotherms, the microporous Ax-21 and MSC-30 show very high surface area of 2257 and $2662 \mathrm{~m}^{2} \mathrm{~g}^{-1}$ respectively, activated charcoal (AC) have a surface area of $483 \mathrm{~m}^{2} \mathrm{~g}^{-1}$ and the fully mesoporous supports CNTs and MESO-C possess the lowest surface areas of 253 and $217 \mathrm{~m}^{2}$ $\mathrm{g}^{-1}$ respectively. 

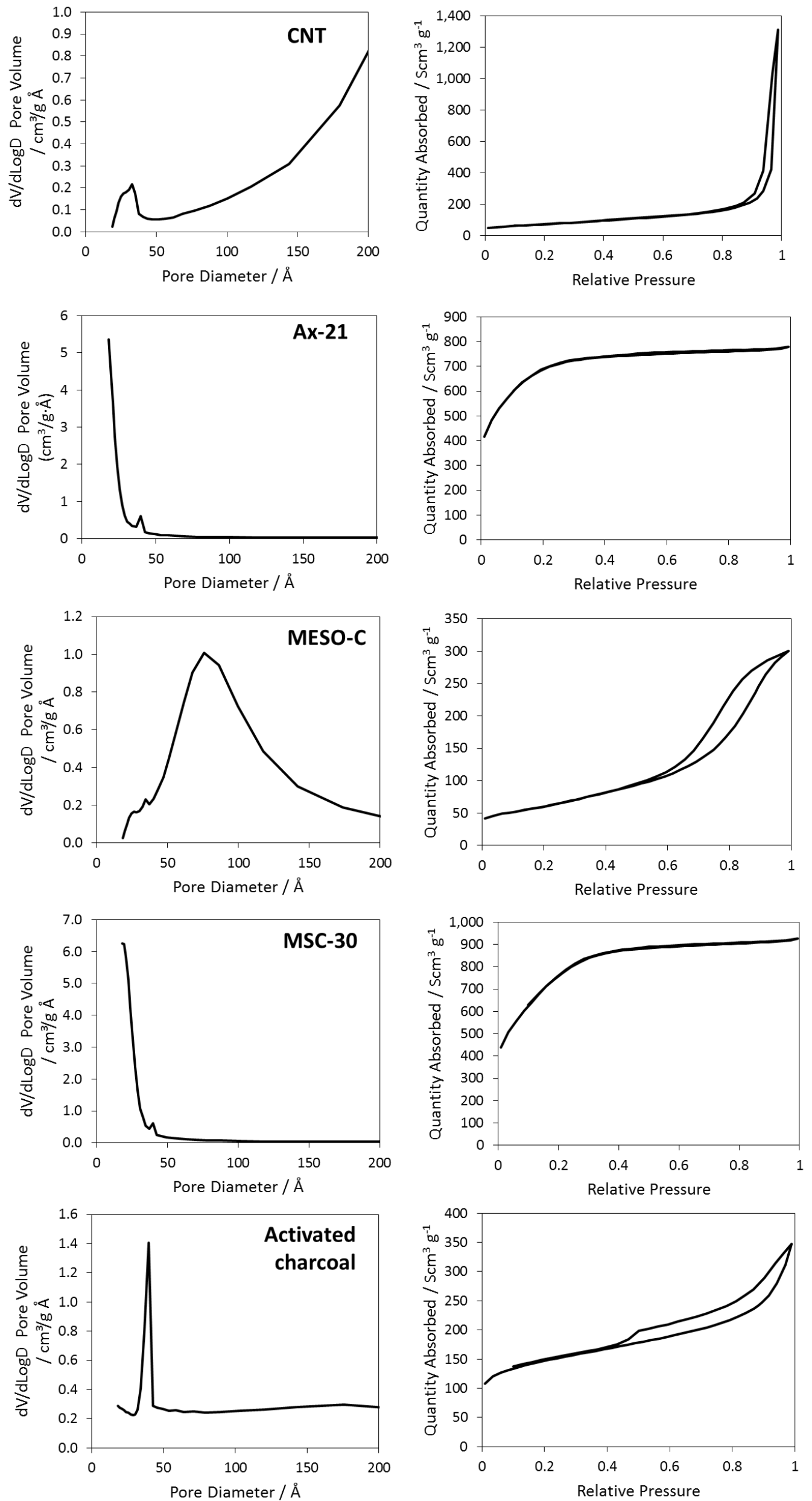
Figure 5: Nitrogen adsorption isotherms at $-196{ }^{\circ} \mathrm{C}$ and corresponding pore size distribution of different carbon materials.

Representative TEM images of cobalt supported on carbon materials catalysts are shown in Figure 6. In all cases, areas of high contrast were observed with a great deal of variability in size. It is important to mention that these areas are not present in the counterpart TEM images of the bare supports, suggesting the formation of cobalt agglomeration up to $20 \mathrm{~nm}$. These potential cobalt agglomerations are in agreement with previous observations where high contrast areas are formed at the edges of the carbon surface during the drying step and also during reduction at high temperatures [28].
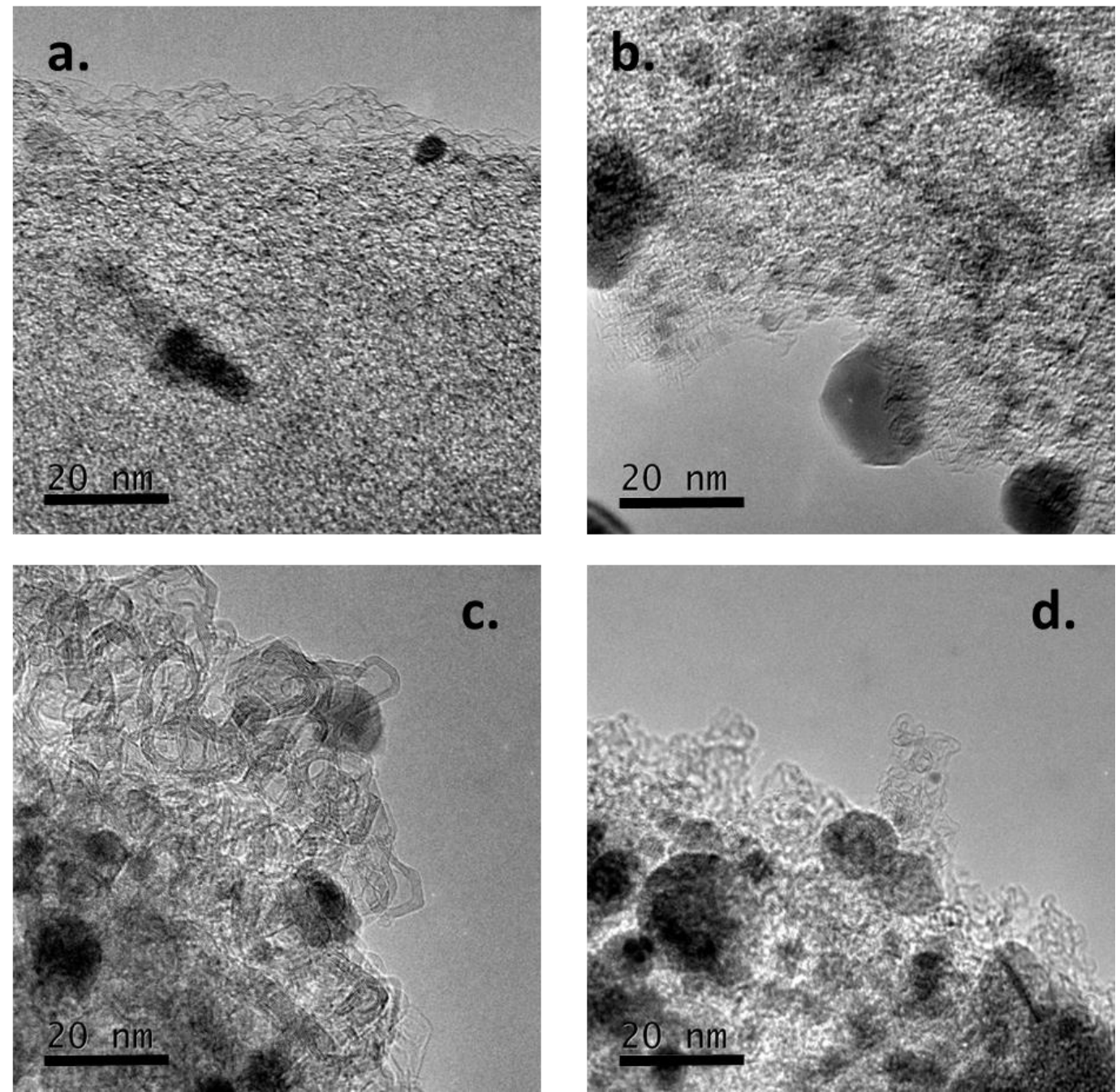

Figure 6: Representative TEM images of 7 wt.\% cobalt supported on a. Ax-21, b. MSC-30, c. MESO-C and d. Activated carbon materials. 
To provide further insights into the distribution of cobalt, energy-dispersive X-ray spectroscopy (EDS) analyses were carried out in the $7 \mathrm{wt} . \% \mathrm{Co} / \mathrm{MSC}-30$, a representative microporous support with pore size of $2.3 \mathrm{~nm}$. The results of which are presented in Figure 7 alongside the TEM image of the same site. The EDS analysis shows that although the dark areas in the TEM image might present a slightly higher cobalt density, cobalt is generally very well dispersed on the catalyst surface and its pore structure. Any cobalt present within the carbon micropores is too small to be resolved from the TEM micrograph and this may well account for a significant proportion of the total cobalt. In addition, the chemical analysis provided a chemical composition of $77.1 \mathrm{wt} . \%$ carbon, $22.4 \mathrm{wt} . \%$ cobalt and $0.5 \mathrm{wt} . \%$ oxygen. The high concentration of cobalt compared to the overall loading of $7.0 \mathrm{wt} . \%$ suggests that the incipient wetness impregnation method employed in this work produced substantial localised variations in metal loading. In addition, it is known that surface chemistry of the carbon materials is closely related to metal dispersion [29], so any variation on the support will be translated into a heterogeneous cobalt dispersion. The small quantity of oxygen suggests that the cobalt was largely in its reduced state after reduction at $400^{\circ} \mathrm{C}$. Although the microscopy results suggest the presence of cobalt nanoparticles with a wide range of sizes, mostly inside the pore structure of the supports, it was not possible to accurately quantify the particle size distribution using this technique. 

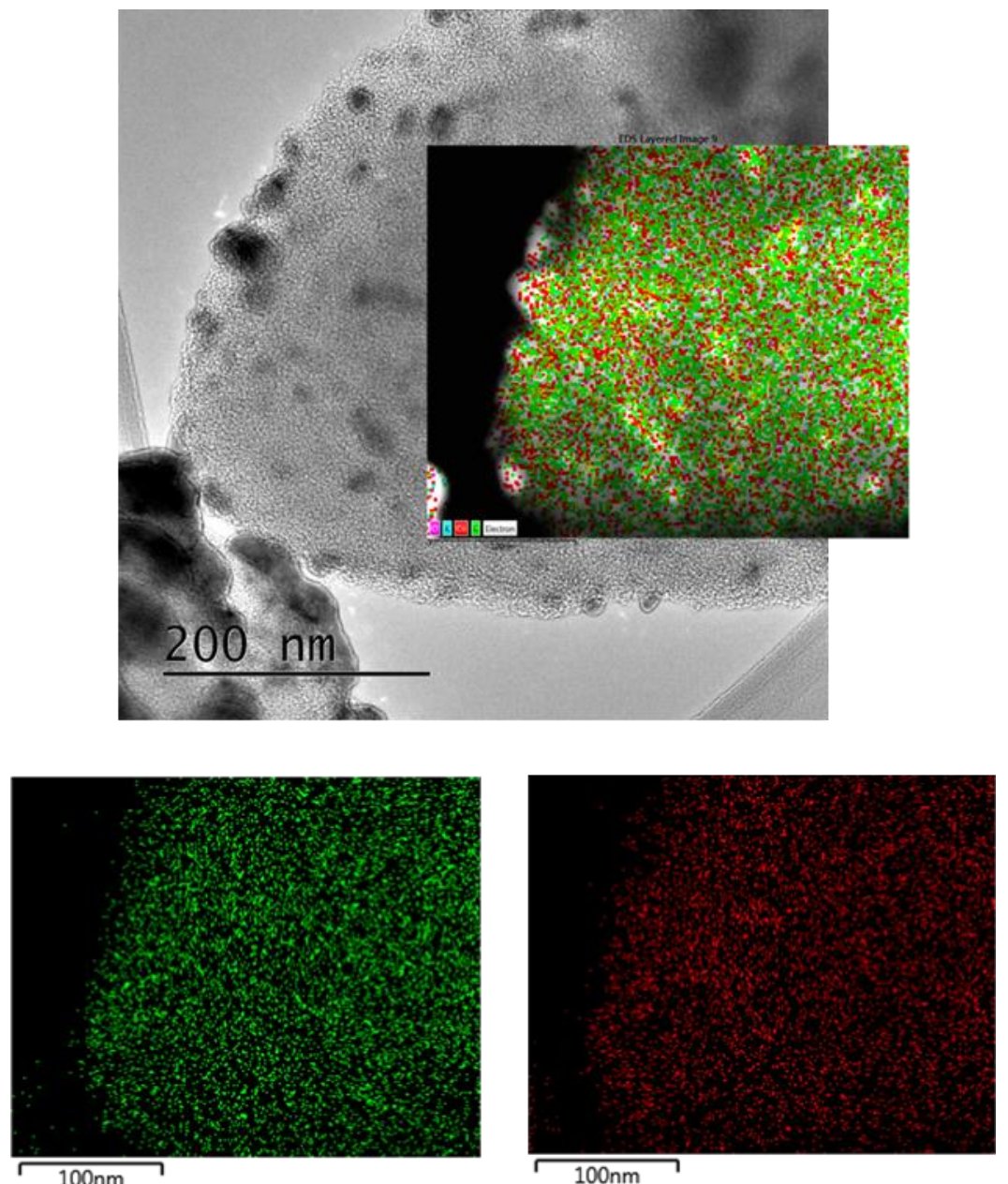

Figure 7: EDS analysis of 7 wt.\% Co on MSC-30. EDS spectra inset from main TEM image where green is carbon, red is cobalt and purple is oxygen.

Powder X-Ray diffraction (XRD) patterns of the different bare carbon materials display

diffraction peaks at $2 \theta$ angles between 10 and $18^{\circ}$ and smaller, broad peaks at $26-29^{\circ}$ and $43^{\circ}$ representative of their low crystallinity. The addition of cobalt to the carbon supports did not produce any significant changes to the spectra, with no visible diffraction peaks corresponding to the cobalt fcc crystal plane or CoO. This observation supports the EDS evidence of highly dispersed cobalt on the supports. Additionally, the larger cobalt agglomeration might not be crystalline enough to show distinguishable diffraction peaks from the carbon background spectra. 
Table 1: Summary of the physical characterisation and catalytic activity for ammonia decomposition reaction of $7 \mathrm{wt} . \%$ cobalt catalysts supported on different carbons.

\begin{tabular}{|c|c|c|c|c|c|c|}
\hline $\begin{array}{c}\text { Carbon } \\
\text { support }\end{array}$ & $\begin{array}{c}\text { Surface } \\
\text { area / } \\
\mathrm{m}^{2} \mathrm{~g}^{-1} \mathrm{a}\end{array}$ & $\begin{array}{c}\text { Pore } \\
\text { size / } \\
\mathrm{nm}^{\mathrm{b}}\end{array}$ & $\begin{array}{c}\text { Raman } \\
\text { Intensity } \\
\mathrm{I}_{\mathrm{D}} / \mathrm{I}_{\mathrm{G}}\end{array}$ & $\begin{array}{c}\text { Apparent } \\
\text { rate of } \\
\text { reaction } \\
\left(425{ }^{\circ} \mathrm{C}\right. \\
* 10^{3}\end{array}$ & $\begin{array}{c}\text { Activation } \\
\text { Energy / } \\
\mathrm{kJ} / \mathrm{mol}\end{array}$ & $\begin{array}{c}\text { Pre- } \\
\text { exponential } \\
\text { factor }\end{array}$ \\
\hline CNT & 253 & 27.5 & 2.6 & 3.76 & 93.57 & $3.81 \mathrm{E}+04$ \\
\hline Ax-21 & 2257 & 2.3 & 3.2 & 11.01 & 88.74 & $5.33 \mathrm{E}+04$ \\
\hline MSC-30 & 2662 & 2.3 & 2.2 & 5.40 & 101.69 & $2.04 \mathrm{E}+05$ \\
\hline MESO-C & 217 & 6.9 & 0.8 & 4.42 & 103.75 & $2.60 \mathrm{E}+05$ \\
\hline AC & 483 & 5.7 & 1.6 & 0.00 & - & 0 \\
\hline
\end{tabular}

${ }^{a}$ Measured by $\mathrm{N}_{2}$ adsorption at $-196^{\circ} \mathrm{C}$ using the BET method from desorption data.

${ }^{\mathrm{b}}$ Measured by $\mathrm{N}_{2}$ adsorption at $-196^{\circ} \mathrm{C}$ using the BJH method with Halsey Faas correction.

Further information of the cobalt particle size and also the cobalt-support interaction can be obtained from temperature programme reduction (TPR) analyses. The normalised results are presented in Figure 8, the fainter line is the TPR profiles of the bare support material and the darker line is the support after impregnation with cobalt. All bare carbon materials with the exception of MESO-C presented a broad peak between 525 and $750^{\circ} \mathrm{C}$; the mesoporous carbon showed very little consumption of hydrogen with a negligible peak intensity compared to the other supports. When $7.0 \mathrm{wt} . \%$ cobalt is added to the different supports, a broad reduction peak appears at around 450 to $480{ }^{\circ} \mathrm{C}$ and the reduction peak at higher temperature corresponding to the bare support is no longer present. In addition to this, a lower reduction temperature peak in the range of $125-300{ }^{\circ} \mathrm{C}$ is observed with cobalt supported on $\mathrm{Ax}-21$, CNTs and activated charcoal. Published studies on cobalt reduction suggest a partial reduction of cobalt $\left(\mathrm{Co}_{3} \mathrm{O}_{4} \rightarrow \mathrm{CoO}\right)$ in the $250-380{ }^{\circ} \mathrm{C}$ range with further reduction to metallic cobalt $(\mathrm{CoO} \rightarrow \mathrm{Co})$ at temperatures between $400-650{ }^{\circ} \mathrm{C}[30]$. It is important to note that the catalysts were in-situ pre-reduced under hydrogen flow at $400^{\circ} \mathrm{C}$ for 3 hours. Interestingly, the only catalysts that did not show apparent catalytic activity was 7 wt.\% Co supported on activated charcoal whose TPR profile suggests a lack of 
cobalt reduction to metallic state during this pre-reduction treatment, although partial reduction to $\mathrm{CoO}$ seems to have taken place at $\sim 285^{\circ} \mathrm{C}$. Consequently, it is suggested that the active form of cobalt is obtained after full reduction [21].

Information about the dispersion of cobalt on the catalyst can be gained by comparing the TPR profile of the bare carbon supports with their counterparts after cobalt addition. The consumption of hydrogen by the bare supports at 525 and $750{ }^{\circ} \mathrm{C}$ is likely to be caused by the methanation reaction occurring by the gasification of surface defects by hydrogen [31] or by reduction of surface oxygen complexes [32]. Both surface defects and surface oxygen complexes are known to act as anchoring points for metal nanoparticles [33], having a direct implication on the final cobalt dispersion across the support. The addition of cobalt considerably reduces or negates the high temperature reduction peak present in the supports which suggests a considerable interaction of the cobalt with such surface groups and a high dispersion. 


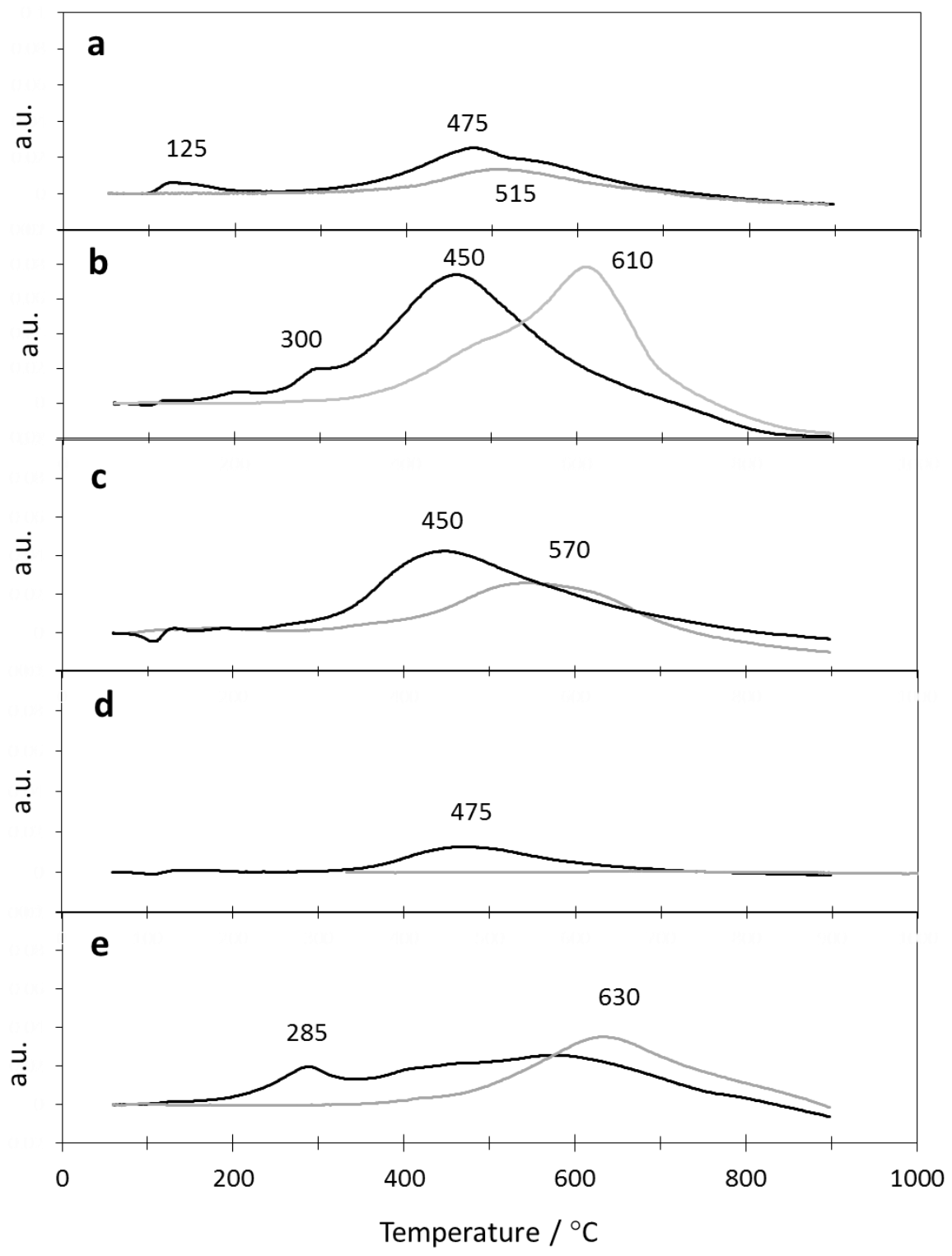

Figure 8: Temperature programme spectra of $7 \mathrm{wt}$ \% cobalt supported on carbon materials a. CNTs, b. Ax-21, c. MSC-30, d. MESO-C and e. Activated charcoal. The fainter line corresponds to the bare support and the darker line after cobalt loading.

The different characterisation analyses reveal that the structure of the carbon support plays a significant role in controlling the dispersion of cobalt. In agreement with previous studies with ordered porous carbons, the pore network of the support not only favours a high cobalt precursor distribution which would result in a high metal dispersion but also can constrain the growth of the cobalt particles inside such pores [28]. Figure 9 shows the relationship between the ammonia decomposition catalytic activity and the average pore size of the different carbon 
supports which presents a clear relationship between microporosity of the carbon support and activity suggesting that small cobalt particles in the range of $\sim 2 \mathrm{~nm}$ size entrapped inside the micropores of the Ax-21 and MSC-30 carbons are highly active in the ammonia decomposition reaction. Indeed, this trend has been further confirmed by analysing the porosity by nitrogen adsorption of the different carbon materials before and after metal impregnation. To provide an example, the surface area of the MSC-30 bare support decreased from $2662 \mathrm{~m}^{2} \mathrm{~g}^{-1}$ to $1842 \mathrm{~m}^{2} \mathrm{~g}^{-1}$ after impregnation with cobalt (7\% wt. Co/MSC-30). In addition, a decrease of the overall pore volume was also observed from $1.23 \mathrm{~cm}^{3} \mathrm{~g}^{-1}$ of the MSC-30 support to $0.85 \mathrm{~cm}^{3} \mathrm{~g}^{-1}$ of the $7 \%$ wt. Co/MSC-30 catalysts while the pore size remain equal to $2.3 \mathrm{~nm}$ in both cases. The decrease in surface area and porosity is also observed, although in less extent, in the mesoporous supports however, in these latter cases, the porous structure does not support the small cobalt particle size $(\sim 2 \mathrm{~nm})$ observed for the microporous carbon catalytic supports.

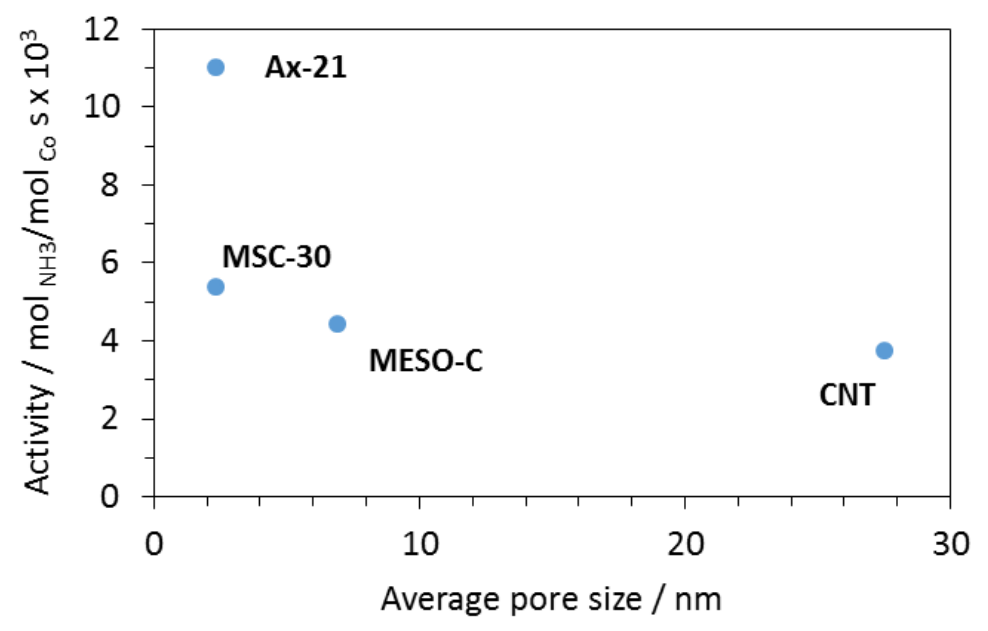

Figure 9: Relationship between ammonia decomposition catalytic activity (at $425^{\circ} \mathrm{C}$ ) of 7 wt. \% Co/carbon catalysts and the average pore size of the carbon support.

These observations provide new guidelines for the design of non-noble metal based catalysts for the production of hydrogen from ammonia, contrary to the ones applicable to rutheniumbased catalysts. While the optimum ruthenium particle size is well known to be in the range 
-3-5 $\mathrm{nm}$ to maximise the concentration of a specific arrangement of five ruthenium atoms, called B5-type sites [10], highly active for the structure sensitive ammonia decomposition reaction [34], this work indicates that the optimum cobalt size is smaller, in the range of $2 \mathrm{~nm}$. This metal dependent optimum particle size has been previously suggested using density functional theory (DFT) simulations. For example, the close packed Ni (111) surface is believed to have a much higher ammonia decomposition activity than the stepped $\mathrm{Ni}(211)$ surface, with a similar configuration to the Ru step sites rich in B5 sites, as the latter is easily blocked by adsorbed nitrogen adatoms [35]. Similar effects are observed for other 3d-late transition metals such as $\mathrm{Fe}$ or $\mathrm{Co}[36]$. Indeed, it has been suggested that $\mathrm{C} 7$ sites present in smaller particles are more active than large crystallites in iron-based systems [37], which seems to be in agreement with the work presented herein.

While the Ax-21 and the MSC-30 carbon supports have a similar average pore size of $2.3 \mathrm{~nm}$, cobalt catalysts supported on the former presents a higher ammonia decomposition activity than a similar system supported on MSC-30. One of the main differences between both microporous carbon materials is their degree of graphitisation; Ax-21 support presents a considerably lower graphitisation degree than MSC-30. As shown in Figure 4, the degree of graphitisation seems to be detrimental to the cobalt-system activity. Again, this is opposite to the trends observed for ruthenium-based systems where CNTs have been shown to be the most active support for ammonia decomposition not only because of its capability of stabilizing optimum sized ruthenium particles but also due to its high graphitisation degree which translated into a high conductivity, facilitates the nitrogen desorption limiting step in the ammonia decomposition mechanism.

To provide further insights of the effect of the electronic modification of the cobalt active sites on their resulting activity, cesium was added to the system as an electron donating promoter. We have recently demonstrated that the addition of cesium to $\mathrm{Ru} / \mathrm{CNT}$ catalysts has 
a similar beneficial electronic effect on the ruthenium active site than an increase of the graphitisation degree of the CNT support [13]. In both cases, the formation of $\mathrm{Ru}^{\delta-}$ active sites are promoted facilitating the nitrogen recombinative desorption on the ruthenium surface. A completely different effect is observed in the cobalt-based catalysts. Figure 10 shows the effect of addition of cesium on the ammonia decomposition activity of various cobalt/carbon catalysts. While the addition of cesium as promoter is detrimental for the catalytic activity of 7 wt. $\%$ Co/Ax-21, it does not seem to affect the activity of the 7 wt.\% Co supported on CNTs or MSC-30 catalysts. The low graphitisation degree of the Ax-21 support seems to be translated into a low electronic modification of the cobalt active sites by the support. In this case, the addition of cesium as an electron donating promoter does modify the cobalt active sites, with an unfavourable effect on its catalytic activity. By contrast, those supports with a higher graphitisation degree such as CNTs and MSC-30 are by themselves capable of electronically modify the cobalt sites, and the addition of cesium does not further affect the activity.
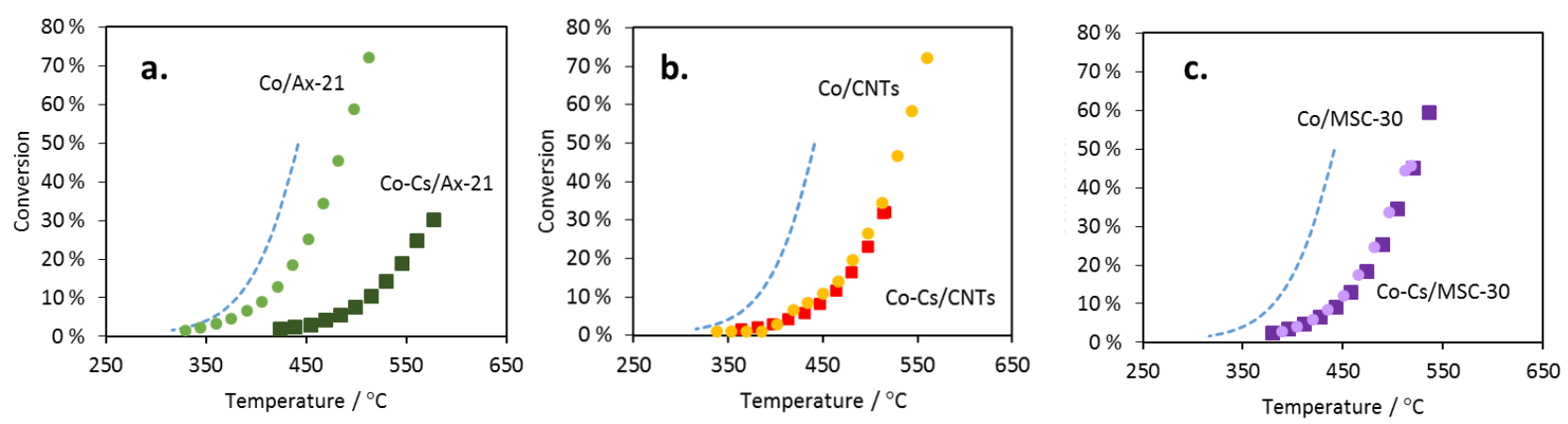

Figure 10: Effect of addition of cesium as electron donating promoter on cobalt-based catalysts supported on carbon. a. Ax-21, b. CNTs and c. MSC-30. Metal loading: 7 wt. \% cobalt and 7 wt. \% cesium. The dashed line in the graphs represents the activity of $7 \mathrm{wt} . \%$ Ru/CNTs as reference. $\bullet$ un-promoted catalysts, $\square$ cesium-promoted catalysts.

These observations provide an explanation of the considerably lower ammonia decomposition activity of the $7 \mathrm{wt} . \% \mathrm{Co} /$ Activated charcoal catalyst despite the presence of some microporosity in the support which is expected to stabilise relatively small cobalt 
nanoparticles. However, its high degree of graphitisation in AC and the consequential interaction with the cobalt particles seems to negate their activity.

\section{Conclusions}

This systematic study of the ammonia decomposition catalytic activity of cobalt catalysts supported on different carbons reveals clear trends between the physical and chemical properties of the supports and the activity of the cobalt sites. It is found that activity is directly related to a high metal dispersion of small $(\sim 2 \mathrm{~nm})$, reduced cobalt nanoparticles stabilised by microporous carbons. Partial cobalt reductions yields negligible activity. In relation to this, electronic modification of the cobalt active sites by the support or by addition of promoters (e.g. cesium) have a detrimental effect on the activity. Indeed, catalytic activity of cobalt is enhanced by carbon supports with a low graphitisation degree. These observations are opposite to the effects observed on ruthenium-based catalysts, providing key information for future development of non-noble metal based catalysts for hydrogen production from ammonia.

\section{Acknowledgments}

The authors would like to acknowledge the UK Engineering and Physical Science Research Council (grant number EP/L020432/2) and the Doctoral Training Centre in the Centre for Sustainable Chemical Technologies at the University of Bath (grant number EP/K016334/1) for funding, and SASOL UK Ltd for supporting TEB's studentship. We would also like to thank the Research Catalysis Group at Harwell ( $\mathrm{RCaH})$ for access to the TEM microscopy facilities.

\section{References}

[1] Hyundai. 
[2] Toyota.

[3] M.P. Suh, H.J. Park, T.K. Prasad, D.W. Lim, Hydrogen Storage in Metal-Organic Frameworks, Chem. Rev., 112 (2012) 782-835.

[4] L. Schlapbach, A. Zuttel, Hydrogen-storage materials for mobile applications, Nature, 414 (2001) 353-358.

[5] A.C. Dillon, M.J. Heben, Hydrogen storage using carbon adsorbents: past, present and future, Appl. Phys. A-Mater. Sci. Process., 72 (2001) 133-142.

[6] R. Lan, J.T.S. Irvine, S. Tao, Ammonia and related chemicals as potential indirect hydrogen storage materials, Int. J. Hydrog. Energy, 37 (2012) 1482-1494.

[7] A. Klerke, C.H. Christensen, J.K. Norskov, T. Vegge, Ammonia for hydrogen storage: challenges and opportunities, J. Mater. Chem., 18 (2008) 2304-2310.

[8] C. Zamfirescu, I. Dincer, Ammonia as a green fuel and hydrogen source for vehicular applications, Fuel Process. Technol., 90 (2009) 729-737.

[9] A. Boisen, S. Dahl, J.K. Norskov, C.H. Christensen, Why the optimal ammonia synthesis catalyst is not the optimal ammonia decomposition catalyst, J. Catal., 230 (2005) 309-312.

[10] F.R. Garcia-Garcia, A. Guerrero-Ruiz, I. Rodriguez-Ramos, Role of B5-Type Sites in Ru Catalysts used for the NH3 Decomposition Reaction, Top. Catal., 52 (2009) 758-764.

[11] J.C. Ganley, F.S. Thomas, E.G. Seebauer, R.I. Masel, A priori catalytic activity correlations: the difficult case of hydrogen production from ammonia, Catal. Lett., 96 (2004) 117-122.

[12] A.K. Hill, L. Torrente-Murciano, In-situ H-2 production via low temperature decomposition of ammonia: Insights into the role of cesium as a promoter, Int. J. Hydrog. Energy, 39 (2014) 7646-7654. [13] A.K. Hill, L. Torrente-Murciano, Low temperature H2 production from ammonia using ruthenium-based catalysts: Synergetic effect of promoter and support, Applied Catalysis B: Environmental, 172-173 (2015) 129-135.

[14] S.F. Yin, Q.H. Zhang, B.Q. Xu, W.X. Zhu, C.F. Ng, C.T. Au, Investigation on the catalysis of COx-free hydrogen generation from ammonia, J. Catal., 224 (2004) 384-396.

[15] A.H. Lu, J.J. Nitz, M. Comotti, C. Weidenthaler, K. Schlichte, C.W. Lehmann, O. Terasaki, F. Schuth, Spatially and Size Selective Synthesis of Fe-Based Nanoparticles on Ordered Mesoporous Supports as Highly Active and Stable Catalysts for Ammonia Decomposition, J. Am. Chem. Soc., 132 (2010) 14152-14162.

[16] H. Zhang, Y.A. Alhamed, A. Al-Zahrani, M. Daous, H. Inokawa, Y. Kojima, L.A. Petrov, Tuning catalytic performances of cobalt catalysts for clean hydrogen generation via variation of the type of carbon support and catalyst post-treatment temperature, Int. J. Hydrog. Energy, 39 (2014) 17573 17582.

[17] H. Zhang, Y.A. Alhamed, W. Chu, Z.B. Ye, A. AlZahrani, L. Petrov, Controlling Co-support interaction in Co/MWCNTs catalysts and catalytic performance for hydrogen production via $\mathrm{NH} 3$ decomposition, Appl. Catal. A-Gen., 464 (2013) 156-164.

[18] Y. Lu, H. Wang, Y. Liu, Q.S. Xue, L. Chen, M.Y. He, Novel microfibrous composite bed reactor: high efficiency $\mathrm{H}(2)$ production from $\mathrm{NH}(3)$ with potential for portable fuel cell power supplies, Lab Chip, 7 (2007) 133-140.

[19] W.Q. Zheng, J. Zhang, Q.J. Ge, H.Y. Xu, W.Z. Li, Effects of CeO2 addition on Ni/Al2O3 catalysts for the reaction of ammonia decomposition to hydrogen, Appl. Catal. B-Environ., 80 (2008) 98-105.

[20] H. Zhang, Y.A. Alhamed, Y. Kojima, A.A. Al-Zahrani, L.A. Petrov, COBALT SUPPORTED ON CARBON NANOTUBES. AN EFFICIENT CATALYST FOR AMMONIA DECOMPOSITION, C. R. Acad. Bulg. Sci., 66 (2013) 519-524.

[21] Z. Lendzion-Bielun, R. Pelka, W. Arabczyk, Study of the Kinetics of Ammonia Synthesis and Decomposition on Iron and Cobalt Catalysts, Catal. Lett., 129 (2009) 119-123.

[22] D. Varisli, N.G. Kaykac, COx free hydrogen production over cobalt incorporated silicate structured mesoporous catalysts, Appl. Catal. B-Environ., 127 (2012) 389-398.

[23] T.E. Bell, L. Torrente-Murciano, H2 production via ammonia decomposition using non-noble metal catalysts - a review, Top. Catal., In press (2016).

[24] S.B. Simonsen, D. Chakraborty, I. Chorkendorff, S. Dahl, Alloyed Ni-Fe nanoparticles as catalysts for NH3 decomposition, Appl. Catal. A-Gen., 447 (2012) 22-31. 
[25] M.C.J. Bradford, P.E. Fanning, M.A. Vannice, Kinetics of NH3 decomposition over well dispersed Ru, J. Catal., 172 (1997) 479-484.

[26] L. Li, Z.H. Zhu, Z.F. Yan, G.Q. Lu, L. Rintoul, Catalytic ammonia decomposition over $\mathrm{Ru} /$ carbon catalysts: The importance of the structure of carbon support, Appl. Catal. A-Gen., 320 (2007) 166-172.

[27] L. Zhang, A.N.C. van Laak, P.E. de Jongh, K.P. de Jong, Zeolites and Catalysis Synthesis, Reactions and Applications, Wiley-VCH2010.

[28] C.X. Zhao, Y.X. Yang, Z.X. Wu, M. Field, X.Y. Fang, N. Burke, K. Chiang, Synthesis and facile size control of well-dispersed cobalt nanoparticles supported on ordered mesoporous carbon, J. Mater. Chem. A, 2 (2014) 19903-19913.

[29] L. Li, Z.H. Zhu, G.Q. Lu, Z.F. Yan, S.Z. Qiao, Catalytic ammonia decomposition over CMK-3 supported Ru catalysts: Effects of surface treatments of supports, Carbon, 45 (2007) 11-20.

[30] N. Fischer, E. van Steen, M. Claeys, Preparation of supported nano-sized cobalt oxide and fcc cobalt crystallites, Catal. Today, 171 (2011) 174-179.

[31] I. Rossetti, F. Mangiarini, L. Forni, Promoters state and catalyst activation during ammonia synthesis over Ru/C, Appl. Catal. A-Gen., 323 (2007) 219-225.

[32] J.S. Lu, Effect of surface modifications on the decoration of multi-walled carbon nanotubes with ruthenium nanoparticles, Carbon, 45 (2007) 1599-1605.

[33] P. Serp, M. Corrias, P. Kalck, Carbon nanotubes and nanofibers in catalysis, Appl. Catal. A-Gen., 253 (2003) 337-358.

[34] W. Rarog-Pilecka, E. Miskiewicz, D. Szmigiel, Z. Kowalczyk, Structure sensitivity of ammonia synthesis over promoted ruthenium catalysts supported on graphitised carbon, J. Catal., 231 (2005) 11 19.

[35] X.Z. Duan, G. Qian, C. Fan, Y. Zhu, X.G. Zhou, D. Chen, W.K. Yuan, First-principles calculations of ammonia decomposition on Ni(110) surface, Surf. Sci., 606 (2012) 549-553.

[36] X.Z. Duan, J. Ji, G. Qian, C. Fan, Y. Zhu, X.G. Zhou, D. Chen, W.K. Yuan, Ammonia decomposition on $\mathrm{Fe}(110), \mathrm{Co}(111)$ and $\mathrm{Ni}(111)$ surfaces: A density functional theory study, J. Mol. Catal. A-Chem., 357 (2012) 81-86.

[37] A. Jedynak, Z. Kowalczyk, D. Szmigiel, W. Rarog, J. Zielinski, Ammonia decomposition over the carbon-based iron catalyst promoted with potassium, Appl. Catal. A-Gen., 237 (2002) 223-226. 